\title{
Effect of light-dark changes on the locomotor activity in open field in adult rats and opossums
}

\author{
I. Klejbor ${ }^{1}$, B. Ludkiewicz ${ }^{1}$, K. Turlejski ${ }^{2}$ \\ ${ }^{1}$ Department of Anatomy and Neurobiology, Medical University of Gdansk, Gdansk, Poland \\ 2Department of Molecular and Cellular Neurobiology, Nencki Institute of Experimental Biology, Warsaw, Poland
}

[Received 4 July 2013; Accepted 6 September 2013]

\begin{abstract}
There have been no reports on how the light-dark changes determine the locomotor activity of animals in the group of high reactivity $(H R)$ and low reactivity $(L R)$. In the present study we have compared selected parameters of the locomotor activity of the $H R$ and the $L R$ groups of the laboratory opossums and Wistar rats during consecutive, light and dark phases in the open field test. Sixty male Wistar adult rats, at an average weight of $350 \mathrm{~g}$ each, and 24 adult Monodelphis opossums of both sexes at an average weight of $120 \mathrm{~g}$ each were used. The animals' activity for $2 \mathrm{~h}$ daily between the hours of 17:30 and 19:30, in line with the natural light-dark cycle were recorded and then analysed using VideoTrack ver. 2.0 (Vievpoint France). According to our results, we noted that a change of the experimental conditions from light to dark involves an increase in the locomotor activity in rats and opossums of the HR group, while there is no effect on the activity of the rats and opossums in the LR group. Locomotor activity in the HR rats, both in the light and dark conditions is characterised by a consistent pattern of change - higher activity in the first stage of the recording and a slowdown (habituation) in the second phase of the observation. The locomotor activity of the opossum, during both light and dark conditions, was observed to be at a consistently high level compared to the rats. (Folia Morphol 2013; 72, 4: 300-305)
\end{abstract}

Key words: opossum, rats, locomotor activity, open field, light-dark change

\section{INTRODUCTION}

Rats (rodents) and opossums (marsupial mammals whose first appearance dates back 100-140 million years ago) are nocturnal animals and the bulk of their activity takes place at night. In the course of evolution, animals developed many adaptations to nocturnal life, such as the acute sense of smell or the ability to dig underground burrows and tunnels to shelter during the day [5, 27-29]. These adaptations were probably a result of moving into new ecological niches to avoid predators.

Besides the anatomical differences (absence of placenta or corpus callosum in the brain), the opossum shows a remarkably different behaviour when compared to other rodents. Opossums expressed a higher rate of locomotor activity, lower latency before exploration of the novel object and had a tendency for choosing a high-risk behavioural strategy as opposed to rats. In general, opossums preferentially use the high-risk exploration strategy while rats mostly rely on defensive behaviour $[2,7,12,13,17]$.

Study done on rodents (such as rats) and opossums showed similar behavioural reactions and enabled us to divide the studied animals into 2 different phenotypic groups, representing 2 opposite behavioural strategies - high reactivity $(H R)$ and low reactivity (LR) group. Animals referred to as highly reactive are characterised by a much higher loco- 
motor activity in comparison to animals with lower activity. Rats from the HR group, along with a higher locomotor activity in a new environment, also have lower levels of anxiety and an increased vulnerability to addiction, as opposed to the rats from LR group $[3,10,11,14-16]$. In the opossum, the level of locomotor activity in a new habitat may reflect the level of general arousal [9, 13].

It is known that for the nocturnal animals light is a factor that influences locomotor activity and can be an aversive stimulus that provokes anxiety-like behaviour $[8,18,19]$, especially in albino animals like Wistar rats $[1,20]$.

There have been no reports on how the light-dark changes determine the locomotor activity of animals in the group of HR and LR. In the present study we compared selected parameters of locomotor activity of the HR and the LR groups of the laboratory opossums and Wistar rats during consecutive, light and dark phases in the open field test, which represents a situation in which the animals can engage in active exploration of a novelty [6].

\section{MATERIALS AND METHODS}

Sixty male Wistar adult rats, at an average weight of $350 \mathrm{~g}$ each and 24 adult Monodelphis opossums of both sexes at an average weight of $120 \mathrm{~g}$ each were used. Having in mind spontaneous locomotor activity, the focus of our study was differentiating the animals into 2 opposite groups - HR and LR. Both, opossums and rats were placed into a special experimental box where we registered the chosen parameters of movement activity. The box was constructed of white plastic with the floor dimensions of $50 \times 50 \mathrm{~cm}$ and a wall height of $50 \mathrm{~cm}$. Above the box, an infrared camera was placed, which registered the entire study. Beneath the floor, an array of infrared light-emitting diodes was placed. They were invisible to the animals, but enabled us to register the animal movements in the darkness. The camera was connected to the computer equipped with a program used for behavioural analysis called VideoTrack ver. 2.0 (Vievpoint France). We registered the animals' action for $2 \mathrm{~h}$ daily between the hours of 17:30 and 19:30, in line with the natural light-dark cycle in such a manner, that the first hour was the last hour of a daytime. After an hour, the light was turned off and the locomotor activity was registered in the dark. The experiments were carried out in such a way that they were synchronised with the animals' circadian rhythm. During the study the following parameters of spontaneous locomotor activity were recorded: (1) the total distance travelled by an animal (in $\mathrm{cm}$ ); (2) per cent of time the animal spent on the locomotor activity; the speed of movement, classified as slow ( 3.0 to $6.3 \mathrm{~cm} / \mathrm{s}$ ) and rapid movements (speed faster than $6.3 \mathrm{~cm} / \mathrm{s}$ ).

As a basis of our study and for the differentiation of animals into HR and LR groups, we used the sum of the total distance travelled during $2 \mathrm{~h}$ of observation measured in centimetres separately for the light and the dark conditions. From the material that was studied, 8 subjects of the HR and LR rats and, in analogy, 8 subjects of the HR and LR opossums were subjected to statistical analysis, thus evaluating the selected parameters of locomotor activity in intervals of $30 \mathrm{~min}$. Statistical analysis was performed using the ANOVA F test, or (in the circumstances where the ANOVA could not be used), the Kruskal-Wallis test was used. In the occasions where significant statistical variations were observed between the groups, post hoc tests were used (for the F test the Tukey and for Kruskal-Wallis the Dunn test). In all calculations, a level of significance was set at $p=0.05$.

\section{RESULTS}

\section{LR rats}

The statistical analysis showed that in the LR group of rats the total distance travelled by one animal $(512.6 \pm 93.1 \mathrm{~cm})$ and the percentage of its activity in relation to the entire time of an experiment $(4.4 \pm 0.8)$ was significantly higher in the first phase of the recording (0-30 min light conditions), than the distance travelled by an animal in the time between 30 and $60 \mathrm{~min}(54.9 \pm 26.1 \mathrm{~cm}$; light conditions). The other compared factors were not statistically significantly different (Fig. 1).

\section{HR rats}

The parameters of locomotor activity in the HR group of rats were as follows: the total distance travelled by one animal in the first phase of the recording was statistically significantly higher $(3221.5 \pm 135.1 \mathrm{~cm})$ in comparison to the distance travelled in the time 30-60 $\min (1941.6 \pm 170.9 \mathrm{~cm}$; light conditions), and 90-120 $\min (1892.4 \pm 157.9 \mathrm{~cm}$; dark conditions) of the observation.

Furthermore, the total distance travelled by the HR rats in the time window $60-90$ min (3191.7 \pm $\pm 371.9 \mathrm{~cm}$; dark conditions) was higher than in the 

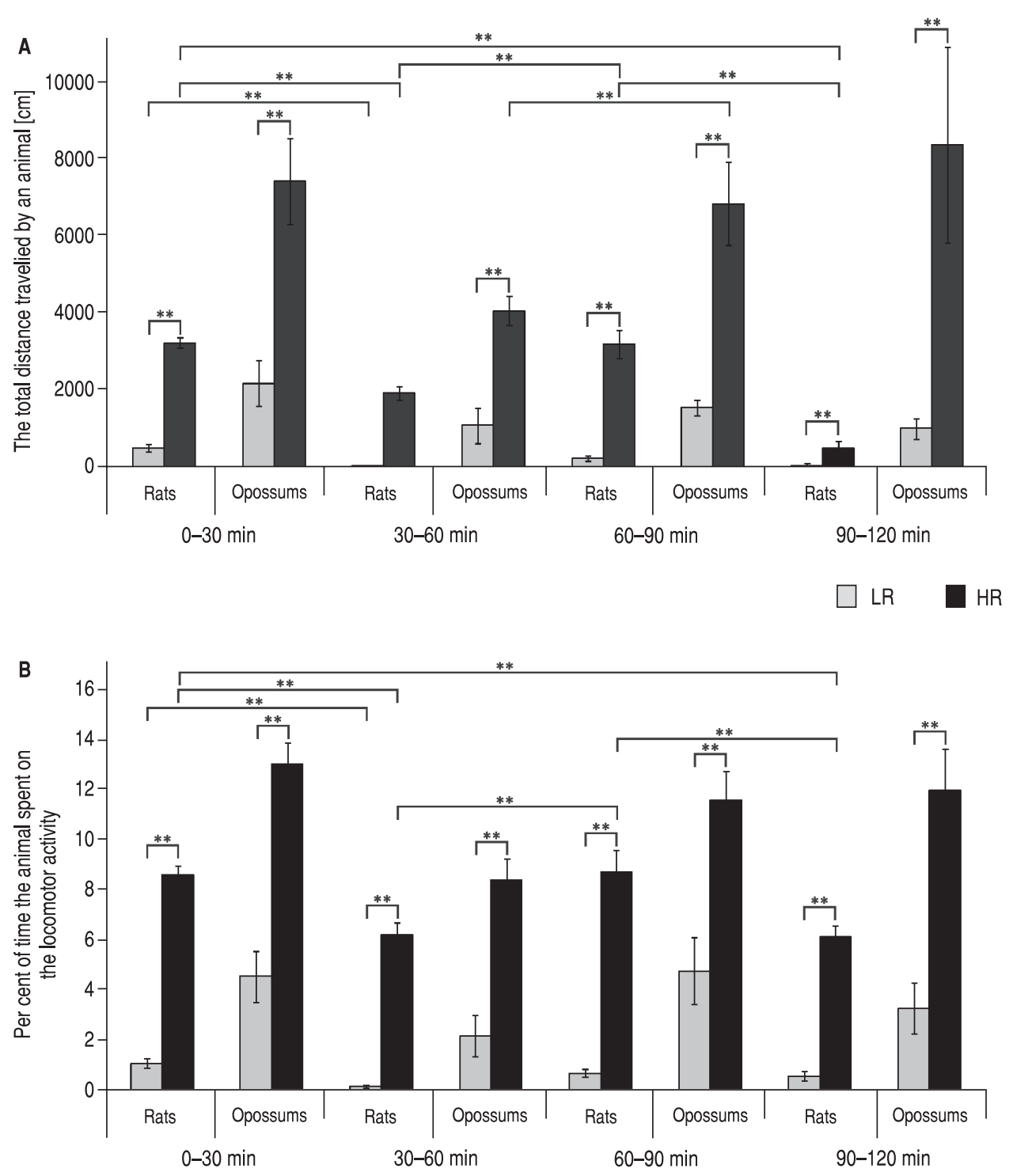

Figure 1. Locomotory activity of the opossums and rats; $\mathbf{A}$. Total distance travelled during the 2-hour session by different group of animals - high reactivity (HR) and low reactivity (LR) (mean \pm SEM); B. Per cent of time the animal spent on the locomotor activity (mean \pm SEM); **p $<0.05$.

time 30-60 $\min (1941.6 \pm 170.9 \mathrm{~cm}$; light conditions) and 90-120 $\min (1892.4 \pm 157.9 \mathrm{~cm}$; dark conditions). The other factors compared were not statistically significantly different.

An analogical scheme of changes was observed in case of differences in the percentage of animal activity. In the HR group, the percentage of activity in relation to the entire time of observation was statistically significantly higher in the first phase of the recording 0-30 $\min$ (34.7 \pm 1.4 ; light conditions), in comparison to the time between 30 and $60 \mathrm{~min}(25.1 \pm$ \pm 1.9 ; light conditions) and the time between 90 and $120 \mathrm{~min}$ ( $24.7 \pm 1.9$; dark conditions) of the observa- tion. Furthermore, the percentage of activity recorded in the time 60-90 $\min (35.1 \pm 3.5$; dark conditions) was significantly higher than in the time 30-60 $\mathrm{min}$ (25.1 \pm 1.9 ; light conditions) and 90-120 $\mathrm{min}$ (24.7 \pm \pm 1.9 ; dark conditions) of the experiment (Fig. 1).

\section{LR opossum}

In the LR group of opossums there have not been any statistically significant differences in all of the considered parameters of motor activity (the total distance travelled by each animal and the percentage of activity in comparison to the entire time of observation) during a different time phase of the recording (Fig. 1). 


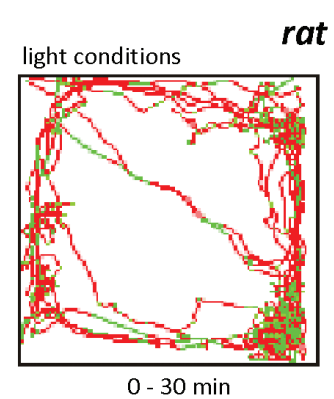

rat $L R$

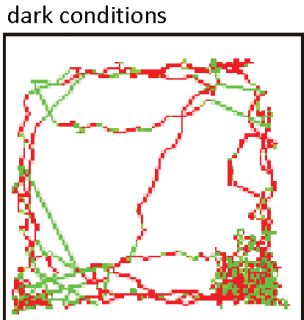

$60-90 \min$

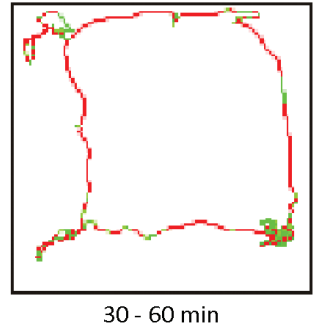

$30-60 \mathrm{~min}$

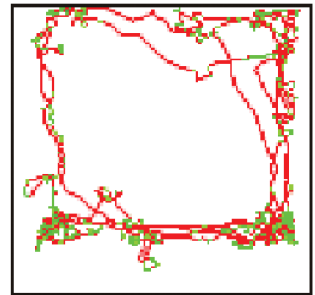

$90-120 \mathrm{~min}$

Drawings of the paths traveled by exemplary rat from LR group.

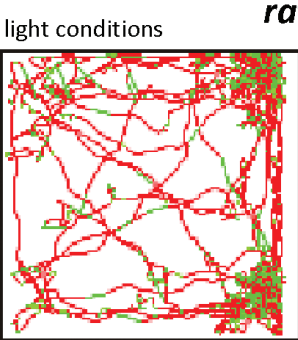

$0-30 \mathrm{~min}$

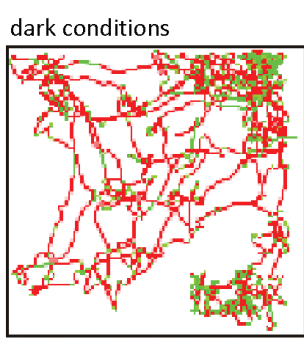

$60-90 \mathrm{~min}$

rat $H R$
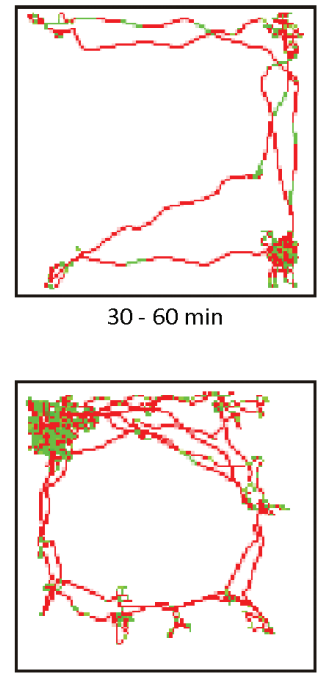

$90-120 \mathrm{~min}$

Drawings of the paths traveled by exemplary rat from HR group.

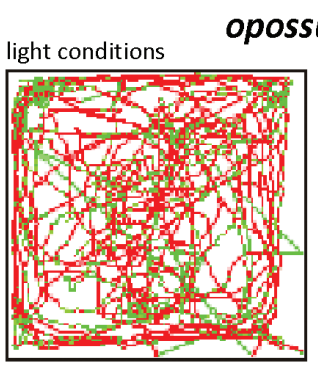

$0-30 \mathrm{~min}$

dark conditions

$60-90 \mathrm{~min}$

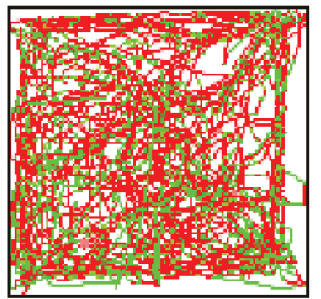

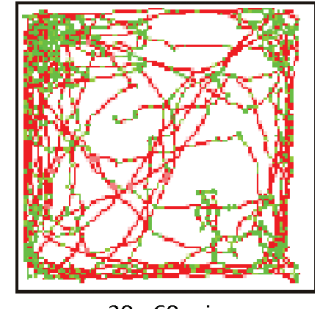

$30-60 \mathrm{~min}$

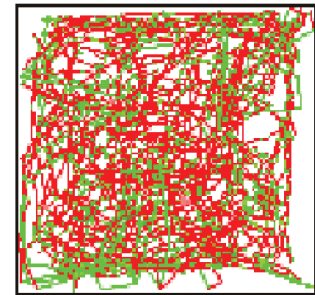

$90-120 \mathrm{~min}$
Drawings of the paths traveled by exemplary opossum from LR group.

light conditions

opossum HR

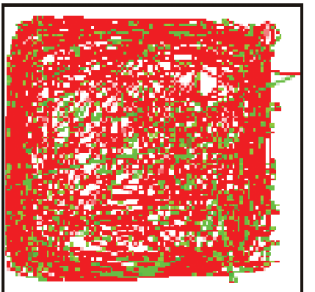

$0-30 \mathrm{~min}$

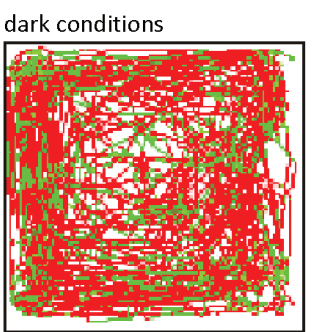

$60-90 \mathrm{~min}$

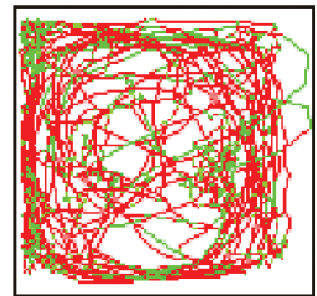

$30-60 \min$

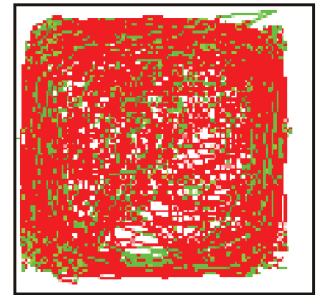

$90-120 \mathrm{~min}$
Drawings of the paths traveled by exemplary opossum from HR group.

Figure 2. Drawings of the total paths travelled by exemplary animals from different groups — high reactivity (HR) and low reactivity (LR); green colour — slow movements $(3.0-6.3 \mathrm{~cm} / \mathrm{s})$; red colour — fast movements $(>6.3 \mathrm{~cm} / \mathrm{s})$.

\section{HR opossum}

In the HR group of opossums there have not been any statistically significant differences in the percentage of activity between different times of the recording. On the other hand, the statistical analysis showed that the whole distance travelled by an HR opossum in the time between 60 and $90 \mathrm{~min}$
(6808.8 $\pm 1064 \mathrm{~cm}$; dark conditions) of the recording was statistically higher in comparison to the distance travelled in time between 30 and $60 \mathrm{~min}$ (4056.4 384.2; light conditions) of the experiment (Fig. 1).

The exemplary trajectories for particular groups of animals are shown on Figure 2. 


\section{DISCUSSION}

Firstly, our results showed that a change of the experimental conditions from light to dark increased the locomotor activity of rats as well as opossums from the HR group, but did not alter the locomotor activity of animals from the LR group. As already described by Piazza et al. [15] and Kabbaj et al. [10, 11], a much more driven exploratory behaviour was the one characterised by rodents included in the HR group, which (despite the typical rat passive-defensive strategy) displayed mainly curiosity and preferred a more active behaviour $[10,11]$. Many authors showed that rats belong to nocturnal animals and such epigenetic factors as light and dark conditions have influenced their level of locomotor activity [4, 21, 22]. Our results showed that the light and dark conditions have a significant effect on locomotor activity in rats of the HR group. Turning off the lights during the observation created more favourable conditions for the active exploration for $\mathrm{HR}$ rats and resulted in the increase of the studied parameters of their locomotor activity. Contrary to that, in the LR group of rats, turning off the lights did not significantly change the studied parameters. The exposition of the LR rats to the new, unfriendly, brightly illuminated environment, makes them fear and much decreases their locomotor activity. An hour that elapsed from the beginning of the more friendly, dark phase, when the light was turned off, was too short period to reduce their level of anxiety. It is probable that our LR group of rats was still anxious in the open field exposure and needed much more time to start a more active exploratory behaviour. Similar findings were described in the opossum, where turning off the lights after registering the parameters resulted in an increase of locomotor activity in the HR group of animals, and they did not change significantly in the LR group. In the HR group, this can be explained by the fact that for these animals every external factor, including turning off the lights, is sufficiently strong of an incentive to encourage a more active exploration. While, for extremely less prone to exploration LR opossum, light/ /dark shift seems too weak stimulation to increase their locomotor activity. This is consistent with the observation that although opossums are the most active during the early hours of the evening, the light is not a limiting factor for their activity [25].

Secondly, our studies showed that the locomotor activity of the HR rats in both, the light and dark conditions was characterised by a consistent pattern of change - higher activity in the first 30 min of the recording and a slowdown (habituation) in the next phase of the recording. The statistical analysis showed that in the light conditions in the HR group of rats the total distance travelled by one animal was significantly higher in the first $30 \mathrm{~min}$ of the recording than the distance travelled by the animal in the time 30-60 min. Furthermore, the total distance travelled by the HR rats in the first phase of the dark conditions (the time window 60-90 min) was higher than in the time 90-120 min. Many studies on exploratory behaviour showed that when an animal explores new surroundings, it exposes itself to harm as for example an attack of a predator, but on the other hand to a profit, an opportunity that the animal may find the shelter or food $[25,26,27]$. Therefore, within one species, intensity will vary depending on the exploratory behaviour, which is associated with the state of the organism's incentive. Węsierska et al. [26, 27] showed that rats use the low risk behavioural strategy and avoid unknown open spaces. In the new open field test, rats are characterised by a strong passive-defensive reaction, which manifests itself in careful behaviours, completely different than that presented by opossums [25-27]. Despite this, in the HR group of rats we observed an increase in active exploration over time. Our results can be explained by the phenomenon called 'habituation' [26, 27]. Habituation is the reflection of a memory formed under the influence of various stimuli received during the exploration [23, 24, 26, 27].

Thirdly, the locomotor activity of the opossum, during both light and dark conditions, was observed to be at a consistently high level compared to the rats. Regardless of the conditions in which the opossums' locomotor activity parameters were recorded, each phase of the opossums behaviour was characterised by high mobility and a variable pattern of exploratory transition from the periphery to the central part of the open field. These observations are consistent with previous reports [21, 25-27] and are closely linked to the biology of opossums, which lead a solitary and predatory lifestyle, and while hunting move fast into an open area. It can be assumed that within a natural biocenosis, such a behavioural strategy was beneficial for them and was recorded by evolution and natural selection. Also, because opossums are domesticated for 
a much shorter period than rats [25], they could be influenced by our observations.

In summary, our studies have shown that under the conditions of continually changing external factors (in this case, the light and dark) in a population as diverse of a species as the opossum and rat, we can distinguish the analogous group of animals characterised by an extremely different locomotor response.

\section{CONCLUSIONS}

1. A change of the experimental conditions from light to dark involves an increase in the locomotor activity in rats and opossums of the HR group, while there is no effect on the activity of the rats and opossums in the LR group.

2. Locomotor activity in the HR rats, both in the light and dark conditions, is characterised by a consistent pattern of change - higher activity in the first stage of the recording and a slowdown (habituation) in the second phase of the observation.

3. The locomotor activity of the opossum, during both light and dark conditions, was observed to be at a consistently high level compared to the rats.

\section{ACKNOWLEDGEMENTS}

This research was supported by the Polish Ministry of Science grant No. 0713/B/P01/2008/34.

The authors wish to thank Dr Dariusz Świetlik for the preparation of the statistical analysis.

\section{REFERENCES}

1. Barker DJ, Sanabria F, Lasswell A, Thrailkill EA, Pawlak AP, Killeen PR (2010) Brief light as a practical aversive stimulus for the albino rat. Behav Brain Res, 214: 402-408.

2. Beardslee $\mathrm{SL}$, Papadakis $\mathrm{E}$, Altman HJ, Harrington GM, Commissaris RL (1989) Defensive burying behavior in maudsley reactive (MR/Har) and nonreactive (MNRA/Har) rats. Physiol Behav, 45: 449-451.

3. Blanchard MM, Mendelsohn D, Stamp JA (2009) The HR/LR model: Further evidence as an animal model of sensation seeking. Neurosci Biobehav Rev, 33: 1145-1154.

4. Cambras T, Vilaplana J, Campuzano A, Canal-Corretger MM, Carulla M, Díez-Noguera A (2000) Entrainment of the rat motor activity rhythm: effects of the light-dark cycle and physical exercise. Physiol Behav, 70: 227-232.

5. Challet E (2007) Minireview: Entrainment of the suprachiasmatic clockwork in diurnal and nocturnal mammals. Endocrinology, 148: 5648-5655.

6. Dubovicky M, Jezova D (2004) Effect of chronic emotional stress on habituation processes in open field in adult rats. Ann N Y Acad Sci, 1018: 199-206.

7. Fadem BH, Corbetta A (1997) Sex Differences and the Development of Social Behavior in a Marsupial, the Gray Short-Tailed Opossum (Monodelphis Domestica). Physiol Behav, 61: 857-861.

8. Fuss J, Ben Abdallah NM, Hensley FW, Weber KJ, Hellweg R, Gass P (2010) Deletion of running-induced hippocampal neurogenesis by irradiation prevents development of an anxious phenotype in mice. PLoS ONE, 5.
9. Geyer MA, Russo PV, Masten VL (1986) Multivariate assessment of locomotor behavior: pharmacological and behavioral analyses. Pharmacol Biochem Behav, 25: 277-288.

10. Kabbaj M (2004) Neurobiological Bases of Individual Differences in Emotional and Stress Responsiveness. Arch Neurol, 61: 1009-1012.

11. Kabbaj M, Akil H (2001) Individual differences in novelty-seeking behavior in rats: a c-fos study. Neuroscience, 106: 535-545.

12. Kalynchuk LE, Gregus A, Boudreau D, Perrot-Sinal TS (2004) Corticosterone increases depression-like behavior, with some effects on predator odor-induced defensive behavior, in male and female rats. Behav Neurosci, 118: 1365-77.

13. Klejbor I, Turlejski K (2012) Different strategies of exploration and phenotypic variability of the locomotor behavior in new environment: Comparative study of the laboratory opossum (Monodelphis domestica) and Wistar rat (Rattus norvegicus). Acta Neurobiol Exp, 72: 452-460.

14. Marinelli M (2005) The many facets of the locomotor response to a novel environment test: theoretical comment on mitchell, cunningham, and mark. Behav Neurosci, 119: 1144-1151.

15. Piazza PV, Deminiere JM, Le Moal M, Simon H (1989) Factors that predict individual vulnerability to amphetamine self-administration. Science, 245: 1511-1513.

16. Piazza PV, Le Moal ML (1996) Pathophysiological basis of vulnerability to drug abuse: role of an interaction between stress, glucocorticoids, and dopaminergic neurons. Annu Rev Pharmacol Toxicol, 36: 359-378.

17. Pisula W, Turlejski K, Stryjek R, Nałęcz-Tolaka A, Grabiec M, Djavadian RL (2012) Response to novelty in the laboratory Wistar rat, wild-captive WWCPS rat, and the gray short-tailed opossum (Monodelphis domestica). Behav Proc, 91: 145-151.

18. Prut L, Belzung C (2003) The open field as a paradigm to measure the effects of drugs on anxiety-like behaviors: a review. Eur J Pharmacol, 463: 3-33.

19. Sarkisova KY, Kulikov MA (2001) Prophylactic actions of the antioxidant agent AEKOL on behavioral (psychoemotional) disturbances induced by chronic stress in rats. Neurosci Behav Physiol, 31: 503-508.

20. Stryjek R, Modlińska K, Turlejski K, Pisula W (2013) Circadian Rhythm of Outside-Nest Activity in Wild (WWCPS), Albino and Pigmented Laboratory Rats. PLoS ONE, 8.

21. Treit $D$, Fundytus $M(1988)$ Thigmotaxis as a test for anxiolytic activity in rats. Pharmacol Biochem Behav, 31: 959-962.

22. Valle FP (1970) Effects of strain, sex, and illumination on open-field behavior of rats. Am J Psychol, 83: 103-111.

23. Van Gaalen MM, Steckler T (2000) Behavioural analysis of four mouse strains in an anxiety test battery. Behav Brain Res, 115: 95-106.

24. Vianna MR, Alonso M, Viola $H$, Quevedo J, de Paris $F$, Furman M, de Stein ML, Medina JH, Izquierdo I (2000) Role of hippocampal signaling pathways in long-term memory formation of a nonassociative learning task in the rat. Learn Mem, 7: 333-340.

25. Węsierska M (2002) Specyfika zachowań eksploracyjnych oposa (Monodelphis domestica) i szczura (Rattus norvegicus) odmiany Long-Evans. Kosmos, 51: 19-33.

26. Węsierska M, Turlejski K (2000) Spontaneous behavior of the gray short-tailed opossum (Monodelphis domestica) in the elevated plus-maze: comparison with Long-Evans rats. Acta Neurobiol Exp, 60: 479-487.

27. Wessierska M, Walasek G, Kilijanek J, Djavadian RL, Turlejski K (2003) Behavior of the gray short-tailed opossum (Monodelphis domestica) in the open field and in response to a new object, in comparison with the rat. Behav Brain Res, 143: 31-40.

28. Wideman C, Murphy H (2009) Constant light induces alterations in melatonin levels, food intake, feed efficiency, visceral adiposity, and circadian rhythms in rats. Nutr Neurosci, 12: 233-240.

29. Yan J, Wang H, Liu Y, Shao C (2008) Analysis of gene regulatory networks in the mammalian circadian rhythm. PLoS Comput Biol, 4. 